\title{
Régulation différentielle de la sécrétion d'antagglutine dans l'épididyme de porc : étude immunocytochimique
}

\author{
Françoise DACHEUX, J.-L. DACHEUX
}

Laboratoire de Physiologie de la Reproduction, I.N.R.A., Nouzilly, 37380 Monnaie, France.

Laboratoire de Physiologie Comparée,

Faculté des Sciences, 37200 Tours, France.

Summary. Differential regulation of antagglutinin secretion in pig epididymis : an immunocytochemical study.

Antagglutinin, a specific protein synthesized by the boar epididymis, was localized in all the principal cells of the initial segment, of the caput and of the corpus but was not detectable in the caudal cells. Castration completely abolished the synthesis and secretion of antagglutin in all the epididymal cells. Androgen treatment led to (1) the restoration of antagglutinin secretion in the caput (middle and distal) and in the corpus, (2) the failure to restore the secretion in the initial segment and in the proximal caput and (3) the emergence of a new secretion of antagglutinin by the caudal cells. These results suggest that (1) antagglutinin is an androgen-dependent protein and (2) the epithelial cells from different segments have differential regulatory mechanisms.

\section{Introduction.}

La maturation des spermatozoïdes apparaît directement liée à l'activité de l'épithélium épididymaire qui sécrète de nombreuses protéines dont certaines sont androgéno-dépendantes (Caméo et Blaquier, 1976 ; Echeverria et al., 1982 ; Brooks, 1983). L'utilisation récente, chez le verrat, d'un marqueur spécifique du fonctionnement de la cellule épididymaire, l'antagglutine (Dacheux et al., 1983) a permis de démontrer la localisation intracellulaire de la biosynthèse, des voies de sécrétion et de transport de cette protéine vers la lumière épididymaire (Dacheux et Dacheux, 1987, 1988).

La présente étude a pour but de déterminer l'androgéno-dépendance de cette protéine. La localisation des sites de synthèse et de sécrétion de l'antagglutine, effectuée tout le long de l'épididyme a été comparée dans trois groupes d'animaux (1) normaux, (2) castrés et (3) castrés supplémentés à la testostérone.

\section{Matériel et méthodes.}

Six verrats adultes: 2 témoins, 2 castrés ( $120 \mathrm{j}$ de castration) et 2 castrés traités à la testostérone $(250 \mathrm{mg} / \mathrm{animal}$, trois fois à $15 \mathrm{j}$ d'intervalle) ont été utilisés dans cette étude. Des échantillons provenant de 9 régions épididymaires 
différentes (Dacheux et Dacheux, 1988) ont été fixés à la glutaraldéhyde, postfixés ou non au tétroxyde d'osmium, déshydratés et inclus dans l'Epon. L'antagglutine a été purifiée à partir du fluide épididymaire caudal collecté par perfusion chez le verrat intact. Les techniques de purification, de préparation des anticorps et l'étude de leur spécificité ont été décrites antérieurement (Dacheux et al.. 1983 ; Dacheux et Dacheux, 1987). Deux techniques immunocytochimiques ont été utilisées: (1) la technique de l'immunoperoxydase sur coupes semi-fines traitées par la méthode à la biotine-streptavidine (Dacheux et Dacheux, 1988) et (2) la technique de l'or colloïdal pour la détection ultrastructurale (Dacheux et Dacheux, 1987).

\section{Résultats et discussion.}

\section{1) Localisation de l'antagglutine chez les animaux témoins (fig. 1.4).}

L'antagglutine a été localisée dans toutes les cellules principales de la tête et du corps (proximal et médian) de l'épididyme avec une sécrétion importante dans la lumière de cet organe. La synthèse qui débute dès les premières cellules du segment initial (fig. 1), augmente dans les cellules de la tête et du corps médian (fig. 4), puis diminue dans le corps distal pour disparaître dans les cellules épididymaires de la queue. Parallèlement, la sécrétion dans la lumière (fig. 4) augmente de la tête à la queue. L'étude ultrastructurale a permis de montrer l'antagglutine dans les différents compartiments intracellulaires: (1) au niveau des sites de synthèse, (2) durant son séjour dans l'appareil de Golgi, (3) durant son transport intracellulaire sous forme de vésicules cytoplasmiques distribuées entre l'appareil de Golgi et l'apex de la cellule et (4) durant sa sécrétion dans la lumière de l'épididyme autour et entre les stéréocils (Dacheux et Dacheux, 1987).

FIG. 1-6. - Localisation de /'antagglutine dans le segment initial (fig. 1 -3) et dans le corps médian (fig. 4-6) de l'épididyme de porcs intacts (fig. 1,4), castrés (fig. 2, 5) et castrés supp/émentés à la testostérone (fig. 3,6). Chez les témoins (fig. 1,4), l'antagglutine est localisée dans tous les appareils de Golgi ( $G$ ) des cellules principales et dans la lumière du tube (L), autour et entre les stéréocils (flèche) tandis que les noyaux $(N)$ et les muscles lisses $(M)$ sont négatifs.

Après castration (fig. 2,5), toutes les cellules principales ( $E$ ), fortement réduites en hauteur et ne présentant plus de stéréocils (flèches) apparaissent complètement négatives avec une zone de Golgi indistincte dans le cytoplasme.

Le traitement à la testostérone est incapable de restaurer, dans le segment initial (fig. 3), la synthèse d'antagglutine. Les cellules principales (E), complètement négatives (fig. 3 ), ressemblent à celles des castrés. Noter seulement la réapparition de courts stéréocils dans la lumière (flèches). Dans le corps de l'épididyme (fig. 6), le traitement à la testostérone restaure la sécrétion de l'antagglutine au niveau de tous les saccules de Golgi (G) et dans la lumière du tube (L), autour et entre les stéréocils réapparus (flèches). Toutes les cellules principales présentent, en outre, de nombreuses vésicules apicales, très riches en antagglutine ( $v$ ) qui déversent directement leur sécrétion dans la lumière du tube (L). ( × 500). 


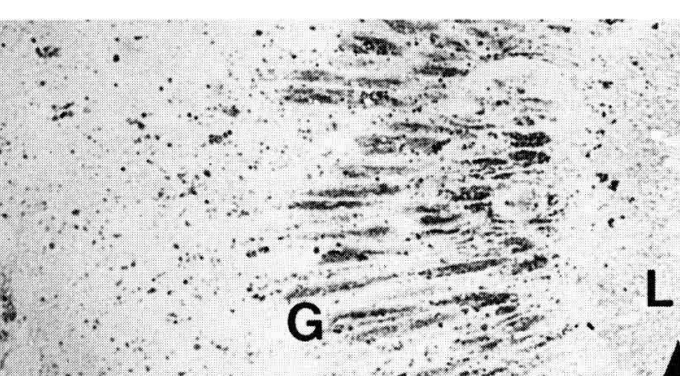

$\therefore \quad-\frac{2}{4} \quad-1$,

1 .
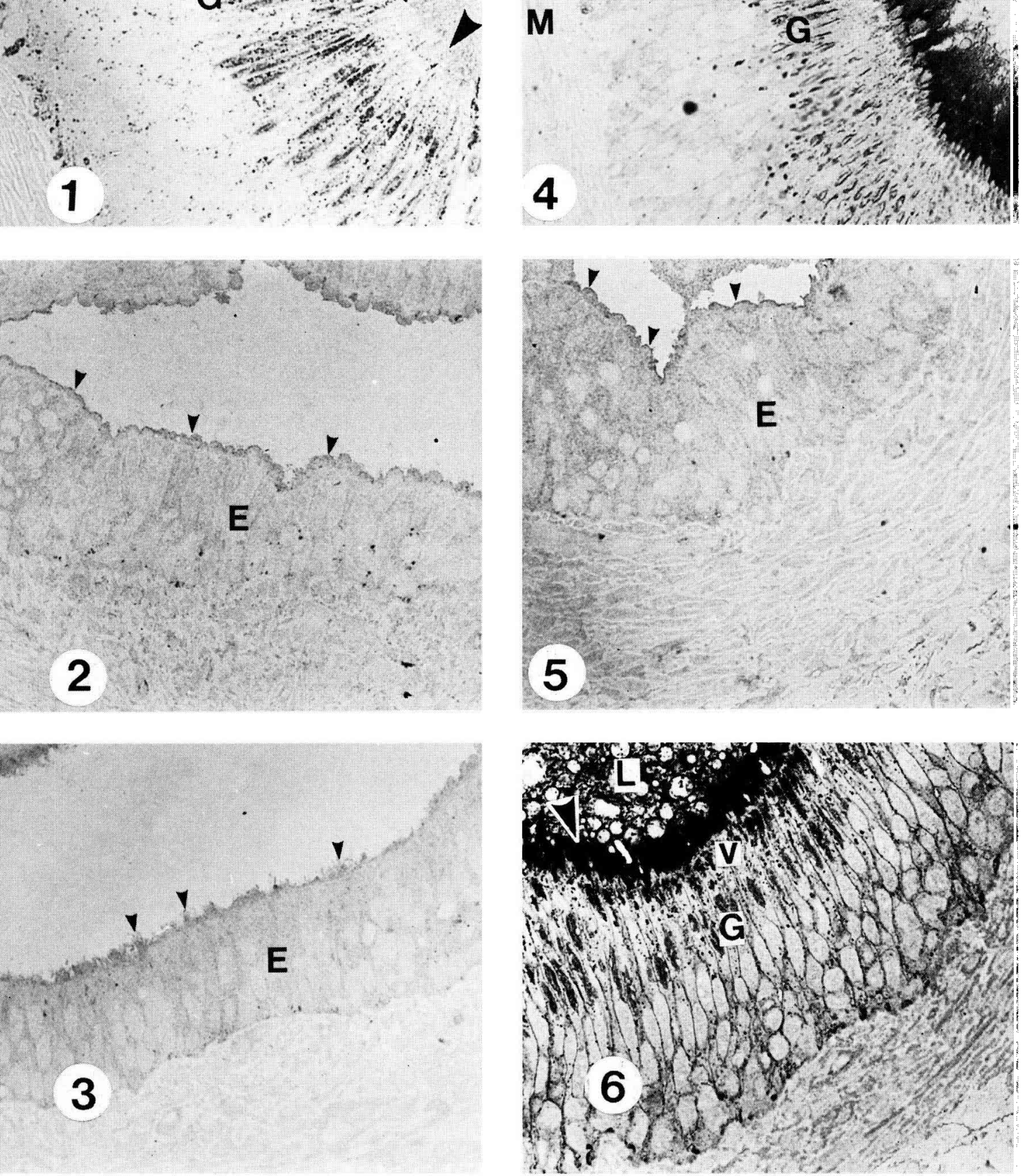


\section{2) Effets de la castration (fig. 2, 5, 8).}

La castration entraîne une régression morphologique (fig. 8) de l'épithélium épididymaire (disparition des stéréocils, diminution de la hauteur des cellules principales, réduction dramatique de l'appareil de Golgi qui devient inactif et difficilement décelable dans le cytoplasme), la perte de la spécificité régionale (disparition des granules denses caractéristiques de la région II) et l'inhibition de la synthèse et de la sécrétion de l'antagglutine dans tous les segments de l'épididyme qui apparaissent complètement négatifs (fig. 2,5).

\section{3) Action de la testostérone chez les castrés (fig. 3, 6, 7, 9, 10).}

L'injection de testostérone chez les castrés restaure la sécrétion de l'antagglutine dans le tube épididymaire. Cependant la réponse est différente selon les régions de l'épididyme. Nous avons mis en évidence trois zones différentes:

a) Une zone réfractaire aux androgènes exogènes (fig. 3). - Le segment initial et la tête proximale restent inaffectés par la testostérone et conservent leur aspect de type «castré ». La sécrétion d'antagglutine n'est pas restaurée dans cette zone.

b) Une zone typiquement androgéno-dépendante (fig. 6, 7, 9, 10). - Dans la tête (médiane et distale) et le corps (proximal et médian), la synthèse et la sécrétion d'antagglutine sont restaurées par le traitement aux androgènes. La reprise de l'activité cellulaire (fig. 6) est marquée par (1) la réapparition des stéréocils dans la lumière et des granules denses dans la zone II, (2) l'extension de la zone supra-nucléaire contenant un appareil de Golgi particulièrement développé et (3) l'apparition d'importantes vésicules apicales (fig. 7). La localisation de l'antagglutine est évidente au niveau de tous les appareils de Golgi et dans la lumière du tube, autour et entre les stéréocils (fig. 6). Toutes les cellules principales stimulées par la testostérone présentent de nombreuses et larges vésicules apicales, très riches en antagglutine qui déversent directement leur produit de sécrétion dans la lumière du tube (fig. 9, 10).

FIG. 7. - Portion apicale de cellules épididymaires de porc castré supplémenté à la testostérone. Noter la présence de nombreuses et larges vésicules apicales (V) se déversant dans la lumière du corps médian de l'épididyme, autour et entre les stéréocils $(S) .(\times 6000)$.

FIG. 8. - Zone identique à celle de la figure 7 mais provenant de porc castré n'ayant subi aucun traitement à la testostérone. Noter la régression morphologique de l'épithélium épididymaire: disparition des stéréocils, diminution de la hauteur des cellules principales avec réduction dramatique de l'appareil de Golgi $(G)$ devenu inactif et réduction de la zone supranucléaire. $(\times 6000)$.

FIG. 9, 10. - Chez les animaux castrés traités à la testostérone, la localisation de l'antagglutine (détectée par la technique de l'or colloïdal) est évidente au niveau des nombreuses vésicules apicales de différente taille (V) (à l'exception des «coated vesicles» figure 9 , flèches), et dans la lumière du tube (L), autour et entre les stéréocils (S). Noter que les particules d'or à l'intérieur des vésicules (fig. 10), sont toujours associées au matériel peu dense (flèches), l'abondance variable disséminée dans la vésicule claire (V). (fig. 9: × $30000 ;$ fig. $10: \times 58000$ ). 


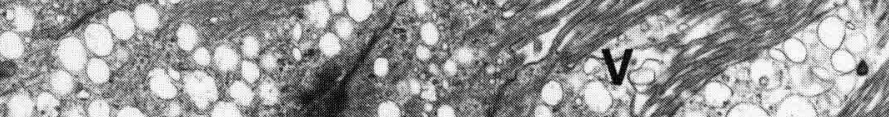

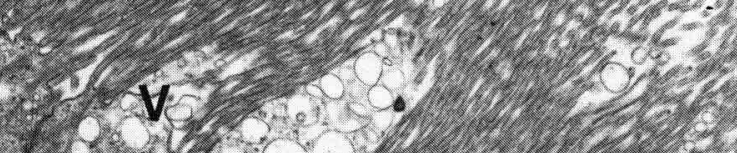

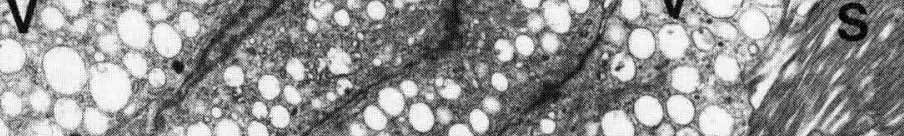

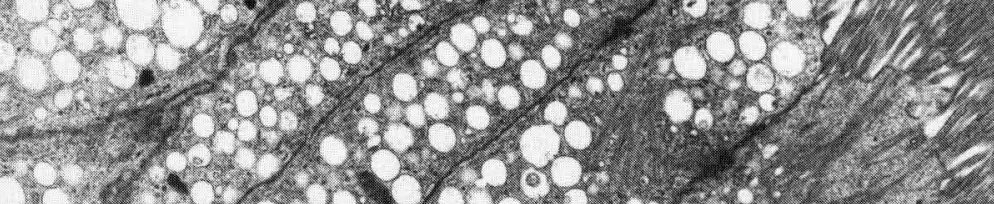

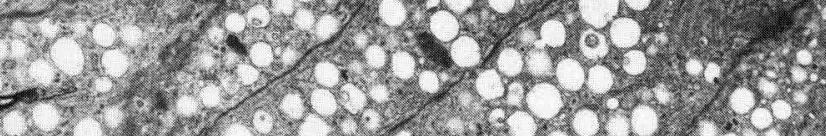

3.

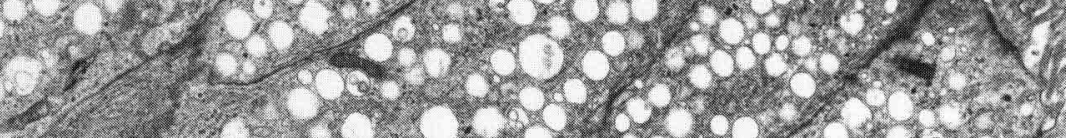

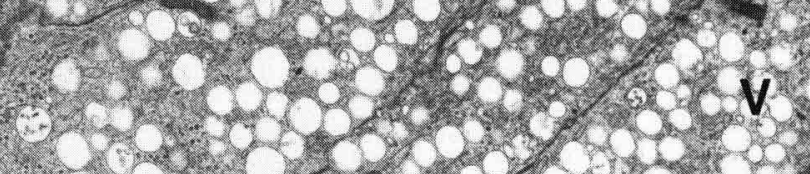

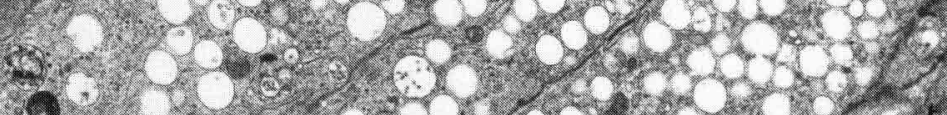

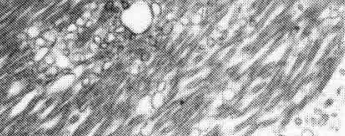

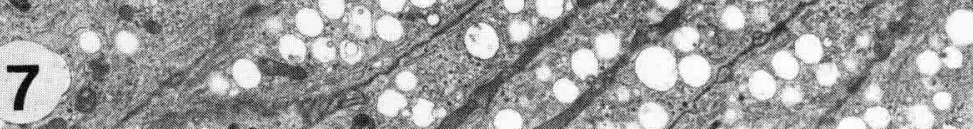

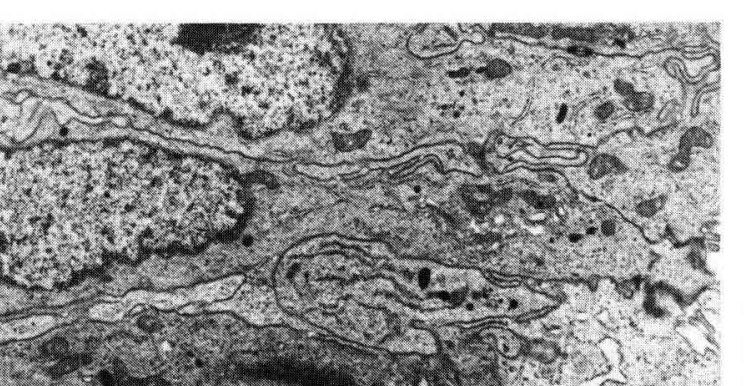

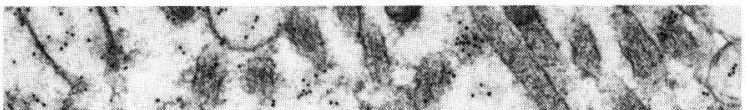
F.

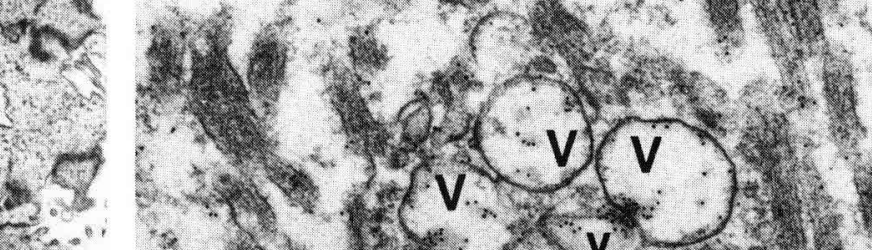
2.7.

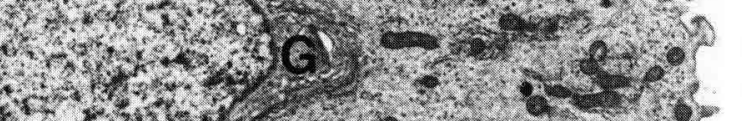

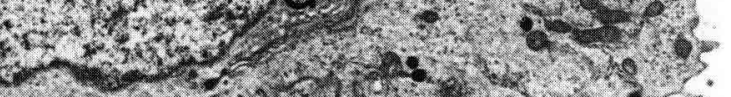

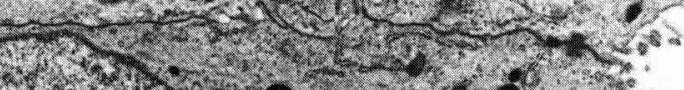

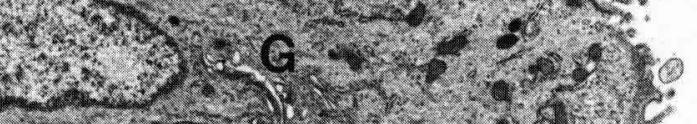

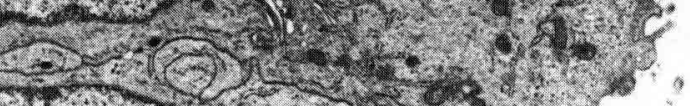

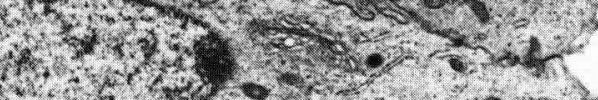

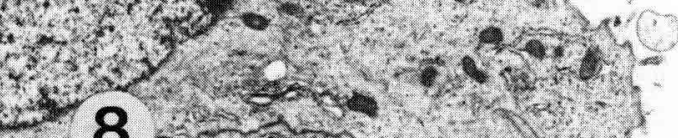
$8 x+2-t$

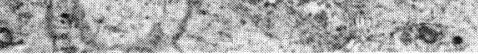

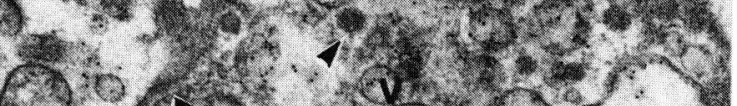

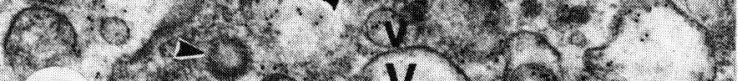

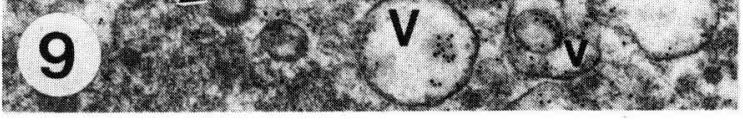

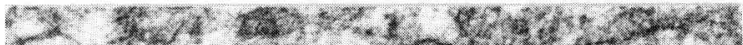

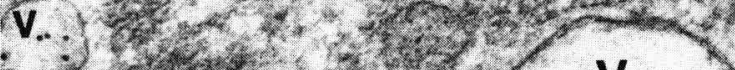

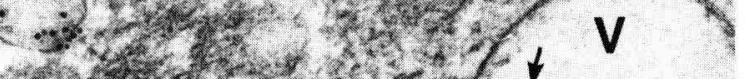

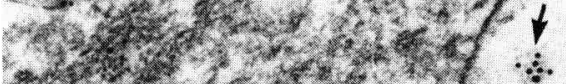

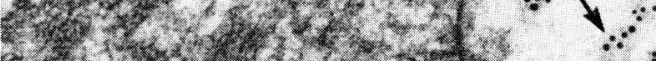

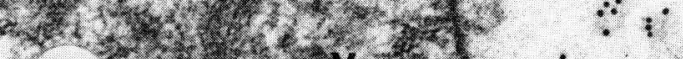

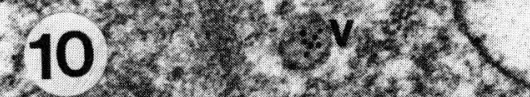

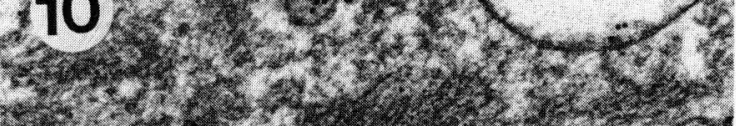


c) Une zone potentiellement capable de sécréter l'antagglutine. La testostérone injectée déclenche la synthèse et la sécrétion d'antagglutine dans les cellules du corps distal et de la queue, zones ne produisant pas d'antagglutine dans les conditions normales. Les cellules principales présentent alors un appareił de Golgi très immunoréactif, beaucoup plus développé que celui des témoins et d'importantes vacuoles apicales déversant l'antagglutine dans la lumière épididymaire.

\section{Conclusion.}

Cette étude a mis en évidence deux points essentiels : (1) l'antagglutine est une protéine androgéno-dépendante et (2) les cellules principales des différents segments épididymaires possèdent une régulation différentielle. Dans la tête (médiane et distale) et le corps de l'épididyme, la sécrétion d'antagglutine est essentiellement contrôlée par la testostérone circulante. Dans les premières zones épididymaires, la sécrétion nécessite la présence de fluide provenant du testicule (androgènes testiculaires et/ou d'autres composés du fluide testiculaire). Au contraire, dans la région caudale de l'épididyme qui apparaît potentiellement capable de sécréter l'antagglutine, la sécrétion semble inhibée chez l'animal normal par certains facteurs qu'il conviendrait d'élucider.

$5^{e}$ Congrès de la Société d'Andrologie de langue française, Paris, décembre 1987.

Remerciements. - Travail subsidié par un contrat INSERM $n^{\circ} 854006$.

\section{Références}

BROOKS D. E., 1983. Epididymal functions and their hormonal regulation. Aust. J. biol. Sci, 36, 205-221.

CAMÉO M. S., BLAQUIER J. A., 1976. Androgen controlled specific proteins in rat epididymis. $J$. Endocr., 69, 47-55.

DACHEUX F., DACHEUX J.-L., 1987. The intracellular pathway of antagglutinin secretion in the boar caput epididymis as revealed by immunogold labelling. Cell Tissue Res., 249, 89-99.

DACHEUX F., DACHEUX J. L., 1988. Immunocytochemical localization of antagglutinin in the boar epididymis. Cell Tissue Res., 252, 329-337.

DACHEUX J.-L., PAQUIGNON M., COMBARNOUS Y., 1983. Head-to-head agglutination of ram and boar epididymal spermatozoa and evidence for an epididymal antagglutinin. J. Reprod. Fertil., 67, 181-189.

ECHEVERRIA F. M. G., CUASNICU P. S., BLAQUIER J. A., 1982. Identification of androgendependent glycoproteins in the hamster epididymis and their association with spermatozoa. J. Reprod., Fertil., 20, 1-7. 